\section{OPORTUNIDAD PARA EL DESARROLLO DE TERRENOS ISLAS EN LA COMUNA DE LAS CONDES}

María Eugenia Pallarés Torres*, Mirtha Pallarés Torres**, Jing Chang Lou***

\section{Resumen}

Se analiza el efecto y potencialidad de la normativa de predio residual destinada a mitigar las consecuencias de la densificación instalada en el año 2003 en el plan regulador de la comuna de Las Condes que, en lo fundamental, está destinada a posibilitar el desarrollo de oportunidades para los predios que presentan superficie inferior a la mínima exigida y que no tienen opción de fusión, porque los entornos inmediatos han sido densificados. El catastro y evaluación de los desarrollos acogidos a la normativa y la detección de predios que podrían asumirla permitió formular una clasificación de oportunidades de gestión y de medidas destinadas a promover la diversidad de opciones

\section{OPPORTUNITY FOR THE DEVELOPMENT OF RESIDUAL LAND IN THE MUNICIPALITY OF LAS CONDES}

María Eugenia Pallarés Torres*, Mirtha Pallarés Torres**, Jing Chang Lou***

\section{Abstract}

This paper analyzes the effects and potential of a regulation intended to mitigate the consequences of a densification process that took place from 2003 within the context of a regulatory plan promoted by the municipality of Las Condes. Such an initiative intends to enable development opportunities for properties smaller than the minimum required and that have no opportunity for merging as the result of the densification of the surrounding environment. According to a survey conducted by this research, the analysis of different developments implemented within the context of this initiative and the identification of lands that may benefit from this provision 
de desarrollo que, respetando las individualidades, incorporan la participación como instancia de control y aseguramiento del equilibrio en la densificación urbana.

\section{PALABRAS CLAVES: EXTERNALIDADES DE LA DENSIFICACIÓN, TERRENOS RESIDUALES, OPORTUNIDADES DE GESTIÓN INMOBILIARIA.}

Recibido: 04-09-2015

Aceptado: 17-05-2016

* Chile. Arquitecto, Universidad de Chile. Doctor, Universidad Politécnica de Madrid. Académico Departamento de Arquitectura, Facultad de Arquitectura y Urbanismo, Universidad de Chile. Correo electrónico:mpallare@uchilefau.cl

** Chile. Arquitecto, Universidad de Chile. Doctor, Universidad Politécnica de Madrid. Académico Departamento de Arquitectura, Facultad de Arquitectura y Urbanismo, Universidad de Chile. Correo electrónico:mipallar@uchile.cl

*** Chile. Arquitecto, Universidad de Chile. Master en Dirección de Empresas Constructoras e Inmobiliarias - MDI, Universidad Politécnica de Madrid. Académico Departamento de Arquitectura, Facultad de Arquitectura y Urbanismo, Universidad de Chile. Correo electrónico: jchang@uchile.cl

172 revista invi 32(89) : 171-196, mayo de 2017 enabling the categorization of management opportunities and measures intended to promote different development opportunities which, by carefully respecting particularities, incorporate participation as a tool for control and to ensure balance within the context of urban densification.

\section{KEYWORDS: EXTERNALITY OF DENSIFICATION, RESIDUAL LANDS, REAL ESTATE MANAGEMENT OPPORTUNITIES}

Received: 04-09-2015

Accepted: 17-05-2016

* Chile. Architect, University of Chile. PhD, Technical University of Madrid. Academic, Department of Architecture at the Faculty of Architecture and Urban Planning, University of Chile. Email: mpallare@uchilefau.cl

** Chile. Architect, University of Chile. PhD, Technical University of Madrid. Academic, Department of Architecture at the Faculty of Architecture and Urban Planning, University of Chile. Email: mipallar@uchile.cl

** Chile. Architect, University of Chile. MSc in Business Administration for Construction and Real Estate Companies MDI, Technical University of Madrid. Email: jchang@uchile.cl 


\section{Introducción}

Presumiblemente la película "Up" producida en el año 2009 por los estudios Disney estuvo inspirada en la historia de Edith Macefield ${ }^{1}$, residente de la ciudad de Seattle que en el año 2006 no cedió a la presión inmobiliaria que pretendía sumar su vivienda al desarrollo de un centro comercial que terminó rodeándola, lo que provocó aislamiento y exclusión, al desvincular la propiedad del nuevo contexto barrial; situación transversal a la mayoría de los centros urbanos generada por los procesos de densificación que implementan los gobiernos locales para organizar el crecimiento y optimizar el uso de los recursos.

Las decisiones de densificación urbana son consecuencia de un determinado estado de desarrollo y de un modo de actuación ante los desafíos de planificación del crecimiento de los asentamientos. Habitualmente estas decisiones obedecen al modelo político-económico imperante y visibilizan el proyecto político y social de la sociedad ${ }^{2}$. La densificación intensifica el uso del suelo a través del aumento en la cantidad de unidades (personas, familias, viviendas, locales, metros cuadrados construidos, etc.), por unidad espacial (predio, lote, manzana, hectárea, etc.); es una estrategia de

1 Martin y Lerman, 2013.

2 Barnett, 1982. ordenamiento urbano caracterizada por la modificación de los parámetros urbanísticos y por operaciones de desarrollo, reconversión de lo existente y mejoras integrales ${ }^{3}$.

En teoría, las medidas de densificación promueven un modelo de ciudad compacto caracterizado por la priorización del transporte público, mayor interacción social en el espacio público ${ }^{4}$, optimización en la dotación de servicios públicos, reutilización de las infraestructuras existentes, conservación de áreas verdes, ahorro de suelo agrícola, promueve un ambiente favorable para los negocios y gobernabilidad menos compleja ${ }^{5}$. Generan externalidades negativas como la expulsión desde las zonas de actuación de los habitantes originales debido al cambio en las condiciones ambientales y en el valor que adquieren los territorios, además de una desequilibrada y habitualmente insuficiente inversión en espacio público producto de la ausencia de condiciones que regulen la producción y ocasión de las dotaciones, asignando prácticamente por omisión la responsabilidad al sector privado, que, sabido es, solo desarrolla los productos financieramente rentables generando desigualdades que provocan rechazo y profundizan la segregación, debido a que las mejores dotaciones se localizan en los sectores de mayores ingresos asegurando la demanda.

\footnotetext{
3 Chavoya, García y Rendón, 2009.

4 López de Lucio, 2000.

5 Chavoya et. al, 2009.
} 
En el tiempo, la sostenida densidad media ${ }^{6}$ de la ciudad de Santiago, que en sesenta años incrementó su superficie casi seis veces y su población cinco veces, sigue siendo más densa que las ciudades de países con altos ingresos y menos densa que las de países con bajos ingresos ${ }^{7}$, comparación mucho más radical cuando se analiza la densidad residencial, que en el caso de Santiago es casi el 60\% de la densidad bruta, mientras en los lugares con mejor calidad de vida urbana varía entre 30\% y $50 \%{ }^{8}$, evidenciando la relevancia de las superficies destinadas a dotaciones urbanas que son las facilitadoras de la vida en comunidad. La Ordenanza General de Urbanismo y Construcción en el artículo 2.1.7., letra f, establece que en el diseño de los planes reguladores intercomunales (instrumento de planificación territorial) se debe estipular la densidad media y/o máxima de las comunas y sus sectores. Normativa que al aplicarse varía debido a la diversidad de parámetros que combina y el

6 La densidad media de la ciudad de Santiago en el año 2002 alcanzó los 85,1 hab/ha, situación semejante a la del año 1940 en que fue de $89,2 \mathrm{hab} / \mathrm{ha}$.

7 Galetovic, 2006.

8 Martínez, 2006. instrumento regulador que aplica, siendo la densidad $^{9}$ el más importante.

Respecto de la densificación ocurrida en la ciudad de Santiago, indicar que morfológicamente ha habido extensión y elevación para acoger más población. Aparentemente, el primer cambio relevante ocurrió a comienzos del siglo XX y contempló la disminución de la superficie predial en las centralidades y la incorporación de los territorios periféricos. Estos cambios pretendían acoger los movimientos demográficos, formalizar las ocupaciones irregulares e integrar a todos los ocupantes del territorio y fueron consecuencia de los cambios políticos, económicos y sociales caracterizados por una mayor inclusión social y una importante intervención en el espacio público que incrementó las dotaciones y visibilizó los atributos de la ciudad ${ }^{10}$.

En el tiempo, y a fin de cubrir la demanda por vivienda, la autoridad administrativa representada por el Ministerio de Vivienda y Urbanismo desplazó el límite urbano, sumando nuevos territorios que permitieron la construcción de viviendas de

9 En las distintas zonas establecidas en los planes reguladores comunales se suelen establecer densidades máximas netas que se calculan respecto de la superficie propia descontando las áreas afectas. Cuando no existe el instrumento se usa la Ordenanza General de Urbanismo y Construcción, que usa como parámetro la densidad bruta calculada sobre la superficie del predio más la exterior hasta el eje del espacio público adyacente existente o previsto en una franja de $30 \mathrm{~m}$. máximo.

10 Pallarés, 2015. 
bajo precio ${ }^{11}$. Las externalidades provocadas evidenciaron las falencias de un proceso de expansión débilmente regulado, que ha entregado al mercado la tarea de hacer ciudad, sin la menor intención de intervención de la autoridad que anticipe los resultados que mayoritariamente profundizan desigualdades en las dotaciones y en la calidad ambiental.

La incorporación de la densificación vertical ocurrida en el siglo XX fue consecuencia de la modernidad y del desarrollo de la técnica que facilitó la edificación en altura, originalmente el objetivo era acoger distintas actividades y liberar el suelo para esparcimiento, expectativa interpretada en acuerdo con la valoración que localmente se otorga a la disponibilidad de espacio público y con los parámetros físicos que condicionan la habitabilidad urbana. Históricamente, la densificación en altura se propone en sectores consolidados con importantes dotaciones, independiente de lo cual es rechazado al ser percibido como una agresión que provoca expulsión y cambio en las condiciones originales de los dominios particulares, por lo que es recomendable profundizar en la internalización de los costos sociales y la construcción de un mercado que pueda manejar las externalidades con eficiencia económica, cohesión social y equilibrio ecológico ${ }^{12}$.

11 Petermann, 2006.

12 Chavoya, et al., 2009.
La extensión de los límites urbanos y los ensanches diseñados a fines del siglo XIX y comienzos del XX en las ciudades europeas, dio paso a la reconstrucción de las centralidades impulsando la densificación vertical ${ }^{13}$, procesos que se consolidaron debido al desarrollo sostenido y constante liderado por el sector público ${ }^{14}$, que incluyó a la ciudadanía ${ }^{15}$. Situación distinta a la historia reciente de la ciudad de Santiago en que la visibilización de proyectos de intervención urbana generó rechazo y propició la organización de la comunidad que apelando a resguardos jurídicos, normativos, técnicos y fundamentalmente mediáticos, logró incluir en los planes mitigaciones compensatorias para las áreas afectadas y los usuarios originales.

Experiencia asumida por los gobiernos locales que consideraron en las propuestas de densificación en altura la posibilidad de rechazo, debido a que por sobre la estimación del intento de entregar a mayor cantidad de ciudadanos los beneficios de los mejores atributos, existe la presunción de que la población original será afectada por el cambio en las condiciones de $\mathrm{uso}^{16}$, siendo necesario regular las acciones a través de medidas de mitigación que integren a los ocupantes originales. Estrategia utilizada en el plan regulador de la comuna de Las Condes que incluyó como castigo para los

\footnotetext{
13 Ballén, 2009.

14 Chavoya, et al., 2009.

15 Ramírez, 2012.

16 Soto y Hormazábal, 2009; Mardones, 2009.
} 
desarrolladores una rebaja en la superficie construible a las fusiones que provocan terrenos residuales o "islas" y estableció una normativa especial para el desarrollo de los predios que adquieren esa condición ${ }^{17}$ permitiéndoles construir productos factibles de acoger distintas sensibilidades.

Considerando que el desarrollo de las intenciones de los planes se construyen en el tiempo, se estimó que a diez años de la instalación de la normativa ${ }^{18}$ especial para predios existentes residuales de densificación era importante analizar su efecto y evaluar la convergencia entre la fundamentación y las expectativas, y conocer el efecto respecto del dominio y de la ciudad, identificando las actuaciones y su integración al paisaje urbano. Enfrentar las expectativas con lo desarrollado permitió evaluar la asertividad de la normativa e identificar potenciales mejoras tendientes a ajustar las condiciones que promuevan la consolidación del instrumento. Destacar que lo importante es el análisis del resultado de la aplicación de la normativa particular que intenta mitigar una situación que, dadas las condiciones jurídicas del país, se seguirá produciendo en todo el territorio cuando se opte por la densificación en altura.

\footnotetext{
17 Artículo 38 Inciso cuarto, quinto, sexto y sétimo, Ordenanza del plan regulador de la comuna de Las Condes.

18 Diciembre 2003.
}

176 revista invi 32(89) : 171-196, mayo 2017

\section{Metodología de análisis}

Para la instalación en el territorio de la normativa del artículo 38 inciso cuarto, quinto, sexto y séptimo de la Ordenanza del plan regulador de la comuna de Las Condes se identificaron las áreas afectas y usando una aplicación de información geográfica se localizaron sobre la planimetría para ser catastradas. El catastro se organizó en tres etapas, primero se revisó la situación del sector registrada en la Dirección de Obras Municipales, luego se constató en terreno y posteriormente se revisó la situación fiscal y de dominio en el Servicio de Impuestos Internos y en el Conservador de Bienes Raíces de Santiago. Con esta información se construyó una ficha por "predio existente residual de densificación", en la que se consignó la situación actual considerando ubicación, superficie predial, dominio, uso, avalúo y tasación comercial.

Los casos se segmentaron según las condiciones que presentaron, separando los que han hecho uso de la normativa y los que podrían hacerlo. Los resultados del estudio se focalizaron en la identificación de posibilidades para el desarrollo de predios existentes residuales de densificación, calculando para cada caso la productividad del predio (cálculo 
de cabida) y se realizó un análisis de mercado, lo que permitió detectar oportunidades de desarrollo. Paralelamente, se revisaron los casos en que se ha aplicado la normativa y la evolución de los productos generados, para conocer el comportamiento de la oferta respecto de variables como destino, productividad, rentabilidad y en los casos más antiguos, plusvalía dada por la segunda o tercera venta. Del enfrentamiento entre ambas situaciones se obtuvieron los parámetros que permitieron construir un listado de oportunidades de desarrollo para los predios que actualmente se encuentran en la condición y un modelo de aproximación a los potenciales casos, además de recomendaciones para una propuesta de ajustes a la normativa destinada a evitar la generación de casos aparentemente sin posibilidad de desarrollo.

\section{Normativa especial para predio existente residual de densificación}

El proyecto de modificación nro.2 del Plan Regulador de la Condes publicado en el Diario Oficial del 5 de diciembre del 2003, definió el término "predio existente residual de densificación" como el existente o fusionado ubicado en sectores de densidad y en áreas de edificación alta o media que no cuentan con la superficie predial mínima establecida y no son susceptible de fusión, debido a que los predios colindantes se encuentran densificados, para luego establecer condiciones especiales de edificación que en lo fundamental mantienen la densidad, aumentan los parámetros de constructibilidad, condicionan la altura en longitud y número de pisos, disminuyen el distanciamiento respecto del antejardín y de los medianeros, y en determinadas condiciones de uso liberan de la exigencia de estacionamientos.

En la fundamentación de la medida se estableció que el objetivo principal era ofrecer opciones de desarrollo para los predios que, a consecuencia de la aplicación de las condiciones de densificación, quedaron fuera del trazado y que era preciso recuperar, fundamentalmente porque el tejido urbano se construye en la diversidad y porque siempre es posible identificar necesidades no cubiertas factibles de acoger en las distintas estructuras disponibles.

El área de aplicación corresponde a los sectores de edificación alta y media (EAa+ca, EAa+cm, EAal, EAa2, EAa4, EAm1, EAaml', EAm2, EAm4) con usos de suelo vivienda y equipamientos e instalaciones de baja y media intensidad (UVO, UV1, UV2, UV3), además de uso comercial para instituciones comerciales y metropolitanas (UC1, UC2). Zonas con coeficiente de constructibilidad entre 1.0 y 4.0, superficie predial mínima entre 800 y 
$2.500 \mathrm{~m}^{2}$, altura máxima entre 14 y 52,5 metros y densidad desde 90 viviendas por hectárea. La potencial área de aplicación es de alrededor de 9 $\mathrm{km}^{2}$ (20\% del territorio comunal urbano) y se localiza en el área de influencia de los principales ejes viales.

Considera dos escenarios: el primero es para todos los usos de suelo, respeta la densidad del área, establece constructibilidad 2.0, cinco pisos y 17,50 metros de altura, rasante $70^{\circ}$, distancia a medianeros 4.00 metros y 5,00 mínimo para antejardín; la segunda opción aplicable en zonas de uso comercial, disminuye la altura a 10,50 metros y tres pisos, para el distanciamiento respecto de los medianeros se refiere a la Ordenanza General de Urbanismo y Construcción (rasante 70\%) y libertad de aplicación para las zonas de edificación continua.

La atracción por habitar la comuna obedece a la valoración que la población hace de los atributos y dotaciones de un sector de la ciudad nacido administrativamente en el año 1901 y que recién en 1920 inició el proceso de urbanización. Es un territorio de $99.40 \mathrm{~km}^{2}$ de los cuales $43,98 \mathrm{~km}^{2}$ corresponden a área urbana, con una población de 205.359 habitantes y 103.794 viviendas, con 290.719 propiedades de las cuales solo $2,2 \%$ están exentas del pago de impuesto territorial ${ }^{19}$, dando cuenta que el avalúo fiscal de un porcentaje muy inferior de viviendas de la comuna ${ }^{20}$ es de $\$ 20.558 .335^{21}$, lo que genera una importante recaudación que incide en el 18,75\% de los ingresos municipales evidenciando la valoración del suelo y sus edificaciones.

La estimación del valor de la localización considera las oportunidades que la ciudad ofrece y que responde a necesidades culturales, siendo frecuentes las asociadas a la disponibilidad de equipamiento y conectividad, atributos reconocidos públicamente en la valorización del suelo urbano ${ }^{22}$, que en el año 2014 instaló a la comuna entre los

19 Sesión Ordinaria $N^{\circ} 10$, Martes 12 de noviembre 2013. Consejo Comunal de Organizaciones de la Sociedad Civil de Las Condes.

206.384 propiedades exentas de impuesto territorial según Sesión Ordinaria $\mathrm{N}^{\circ} 10$, martes 12 de noviembre 2013. Consejo Comunal de Organizaciones de la Sociedad Civil de Las Condes. Corresponde a viviendas, recintos de culto, educacionales y deportivos, Solo las propiedades con uso habitacional, deportivo, educacional o de culto pueden quedar exentas del pago de impuesto territorial.

21 Valor al segundo semestre 2014 que equivale a US\$33.382 (para la conversión se consideró el valor del dólar de la época).

22 Servicio de Impuestos Internos. Re avalúo de bienes no agrícolas 2014. 
lugares con mayor valor por unidad de superficie del país, lo que también se reflejó en los precios que alcanzaron las transacciones de bienes inmuebles, que en promedio superaron en casi tres veces el promedio de venta en la Región Metropolitana de Santiago ${ }^{23}$.

La mayor valoración que alcanzan las propiedades de la comuna respecto de otras ubicaciones refleja los beneficios del lugar valorados por la demanda, y que explican la oposición de algunos sostenedores de dominio al cambio que representa la densificación, que altera la morfología y agrega usuarios modificando el modo de ocupar el territorio, lo que resulta agresivo y muchas veces no deseado ${ }^{24}$. Independiente de los beneficios pecuniarios que reporta el aumento de la productividad del suelo, aparentemente la pérdida del entorno conocido es la mayor dificultad que provoca expulsión, destino que al no ser aceptado genera excepciones transformándose en exclusividades y símbolos del rechazo a la densificación, son predios destinados a la obsolescencia y abandono que la normativa de predio existente residual de densificación acogió permitiendo reinstalar dominios y desarrollar productos integrados al tejido urbano.

23 Comentario validado por el análisis de precios de transacciones de bienes inmuebles nuevos y usados obtenidos desde el Registro de Propiedad del Conservador de Bienes Raíces de Santiago para los años 2010 a 2014.

24 López, Arriagada, Gasic y Meza, 2015.
Los predios residuales son consecuencia de la densificación, por lo que independiente de la potencial área de aplicación la normativa solo es aplicable en las zonas en que el proceso densificatorio está consolidado, por lo que se trata de oportunidades temporales en que la observación del medio resulta fundamental, no solo para el sector privado como principal impulsor del desarrollo y hacedor de proyectos, sino que para la administración del territorio que puede anticipar la ocurrencia e identificar los posibles terrenos residuales que generará la fusión de predios, lo que debiera posibilitar acciones tendientes a evitar conflictos urbanos que en determinadas circunstancias la normativa no logra superar satisfactoriamente, debido a que las condiciones de edificación dificultan la reconversión de los predios o por que la superficie y la morfología permiten levantar volúmenes con escasas posibilidades de ocupación, perjudicando al sostenedor del dominio que no solo es expulsado sino que también sufre una importante pérdida patrimonial, lo que también afecta al sector debido al potencial abandono o del mal uso que provoca deterioro. 
FIGURA 1: PLANO DE LA COMUNA DE LAS CONDES SEÑALANDO LAS ZONAS DE APLICACIÓN DE LA NORMATIVA DE PREDIO EXISTENTE RESIDUAL DE DENSIFICACIÓN E IDENTIFICACIÓN DE LOS PREDIOS

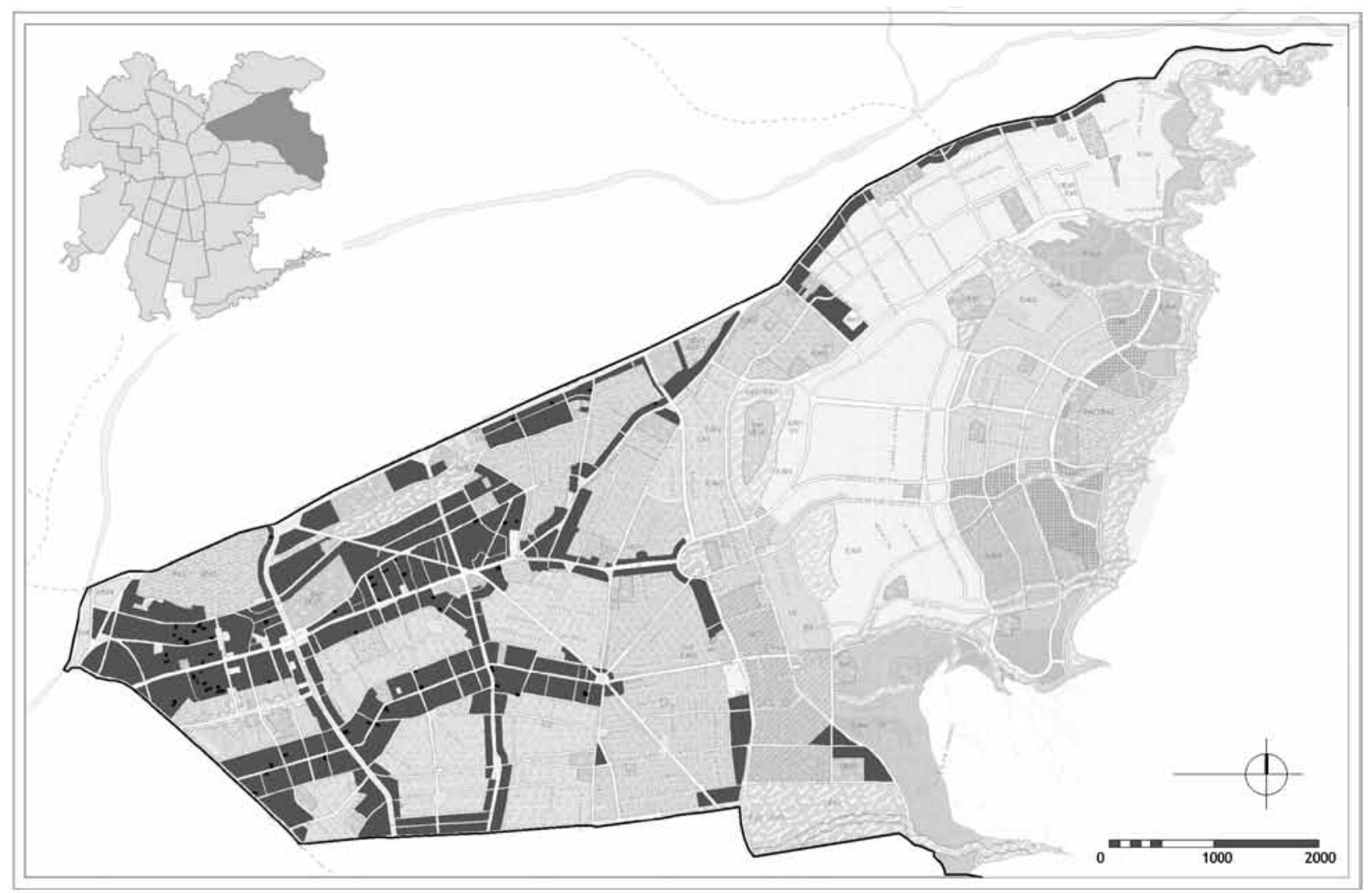

Fuente: Plano de la comuna de Las Condes disponible en https://www.lascondes.cl/informacion_comunal/plan_regulador.html intervenido por los autores. 2014. 


\section{REGISTRO DE PREDIOS EXISTENTES RESIDUALES DE DENSIFICACIÓN}

La densificación en la zona de aplicación de la normativa se encuentra parcialmente consolidada preferentemente sobre los ejes viales más importantes, en sectores comerciales y en las zonas habitacionales que originalmente contaban con mayor superficie predial, evidenciando que en la producción de la densificación en altura se conjugan al menos dos aspectos, el primero asociado con la demanda que prioriza la valora los atributos de localización dados por la conectividad y la dotación de equipamiento y el segundo referido al productor, que opta por las posibilidades más económicas de generación de suelo, habitualmente caracterizado por la menor cantidad de fusiones que aseguran homogeneidad en el precio y velocidad en la gestión.

La evolución del proceso de densificación depende principalmente del sector vivienda y está relacionado con la producción inmobiliaria que varía en consideración con las expectativas de crecimiento del sector, y aunque los volúmenes de venta en la comuna se mantienen constantes con una cuota de participación en la ciudad, que varía entre 6\% y $8 \%{ }^{25}$ y con ingresos entorno al $11 \%$, los precios tienden al alza debido a que las cantidades ofertadas dependen de las oportunidades para el desarrollo de proyectos, incidiendo especialmente la disponibilidad y valor del suelo asociado a la productividad que le asigna la normativa y a las expectativas de los sostenedores del dominio, que valorizan sus bienes en función de la pérdida que significa transferir la propiedad y el entorno que los acogió.

Influye en el desarrollo de proyectos inmobiliarios el tamaño de los emprendimientos, que en estas localizaciones alcanzan montos de inversión y tiempos de desarrollo mayores que en otras ubicaciones, aumentando el riesgo financiero ${ }^{26}$, ya que el volumen factible de desarrollar requiere de importantes superficies que demandan intensas y lentas gestiones que muchas veces desmotivan a los desarrolladores, independiente de la seguridad del retorno financiero, lo que se observa en la cantidad de permisos de edificación otorgados en la serie de tiempo 2008 - $2014^{27}$ en que solo alrededor del 30\% correspondieron a obra nueva.

25 En la ciudad de Santiago la venta anual de viviendas durante los últimos diez años promedio 30.000 unidades con extremos entre 23.000 y 37.000 , media calculada sobre datos publicados por la Cámara Chilena de la Construcción y las consultoras Collect S.A. y Tinsa Chile S.A.

26 Larrea, 2005.

27 Se usaron los datos disponibles en https://www.lascondes.cl/ transparencia/actos_permisos_edificacion.html 
El trabajo de campo realizado durante el segundo semestre del año 2014 permitió identificar 118 predios con superficie inferior a la mínima exigida rodeados de suelos densificados, 56 presentaron destino habitacional por lo que se entendieron como potenciales oportunidades para acoger la normativa, el resto de los predios presentaron cambio de destino con algunos casos acogidos a la normativa especial de predio existente residual de densificación y siete proyectos de obra nueva, cinco con destino vivienda.

Pareciera que la escasa adopción de la normativa obedece a que depende de la disponibilidad de predios generados por proyectos de densificación en altura, lo que inicialmente ocurrió en las zonas de destino comercial y de servicios, y posteriormente en los sectores de destino habitacional, que es donde ha habido mayor resistencia y por consiguiente se han generado predios residuales. También es preciso considerar el desconocimiento respecto de una normativa prácticamente única en el país y también a la resistencia de algunos ciudadanos que al intentar jurídicamente detener el primer proyecto de obra nueva acogido a la normativa, desmotivaron a potenciales desarrolladores que asumieron el reclamo como una amenaza de "no venta".

En el estudio de casos utilizado para identificar escenarios y oportunidades se registró la situación legal de los predios, independiente del uso que presentan, fundamentalmente porque el destino es una condición fiscal que regula los tributos y constituye una obligación para los sostenedores del dominio. Las posibilidades para cada uno los predios se analizaron morfológica y comercialmente a fin de establecer un modelo destinado a orientar las decisiones de aplicación y sugerir mejoras destinadas a potenciar el efecto.

\section{PRODUCTOS ACOGIDOS A LA NORMATIVA}

Tal y como el legislador lo previó, los escenarios de aplicación de la normativa de predios existentes residuales de densificación ha sido acogida en el tiempo con distintos resultados, siendo la más recurrente el cambio de destino, probablemente debido a que existen más oportunidades para la instalación de productos, y porque inicialmente los predios factibles de acoger la normativa se generaron en las zonas con demanda por equipamiento. Los proyectos de obra nueva han tenido un lento desarrollo, siendo los últimos años los más significativos (2012-2014) con una producción del 50\% de los casos.

Una de las particularidades que favoreció al cambio de destino fue la diversidad de opciones de superficies prediales que variaron entre $233,90 \mathrm{~m}^{2} \mathrm{y}$ $1.337 \mathrm{~m}^{2}$, permitiendo acoger distintos programas de equipamiento y servicios, por lo que pareciera que lo relevante fue identificar el tipo de producto 
que mejor se adaptó a las condiciones de origen. En el cambio de destino se acogieron demandas específicas buscando las mejores posibilidades de ocupación de las estructuras existentes, principalmente fue una reconversión de espacialidades caracterizada por la búsqueda de la mejor opción que equilibró posibilidades estructurales y potencial demanda, o sea lo que el continente permitió y el contenedor soportó.

Existen casos en que la normativa permitió formalizar administrativamente situaciones anteriores a la densificación, como el caso del Restaurante Bali Hai, que desde hace más de treinta y cuatro años se localizó en la Avda. Cristóbal Colón en un predio de casi $800 \mathrm{~m}^{2}$ que en el tiempo se transformó en predio existente residual de densificación, debido a que no se sumó a los proyectos del sector porque priorizó la valoración y posicionamiento de la marca en el lugar que resulta difícil de trasladar a otra localización con la misma rentabilidad.

Las adaptaciones para nuevos destinos dieron cuenta de la valoración de las arquitecturas y recogieron las características de localización expresadas en atributos como necesidad, cercanías, vecindad y fundamentalmente comodidad, lo que evidenció que los individuos, por sobre valores urbanísticamente transversales, optan por cuestiones circunstanciales y mayoritariamente individua$l_{e}{ }^{28}$, demostrando que en la medida en que la oferta de servicios responda a sus necesidades, la forma y modo como se localizan es indiferente.

Los destinos de equipamientos de cercanía más exitosos y de fácil adaptación a los distintos escenarios morfológicos y de localización son salas cunas, jardines infantiles, peluquerías, lugares de expendio de comida a domicilio y atención veterinaria, destinos en los que la característica principal es el tamaño del proyecto, es decir la cuantificación de la demanda a la que servirá, debido a que comercialmente no es aconsejable sobredimensionar la oferta. La mejor evidencia de esta consideración la reportaron las instalaciones de pequeñas sedes de servicios destinadas a cubrir acotados territorios, la estrategia que las avala se basa en que en determinados segmentos las personas no están dispuestas a esperar, por lo que la oferta debe ser personalizada, lo que se traduce en muchas opciones a breves distancias, de manera de asumir pequeñas cuotas de mercado en pequeños territorios satisfaciendo la necesidad desde la particularidad, lo que ocurre en el sector con ofertas adecuadas a la capacidad y disposición de pago, y a la escasez de opciones alternativas.

28 No existe un número significativo de denuncias por instalación, permiso o patente comercial de equipamiento de servicio en barrios residenciales y la demanda por ellos es superior a la capacidad instalada: caso de jardines infantiles y salas cunas, restaurantes, peluquerías y veterinarias comprobadas en terreno. 
Hubo situaciones en que las características morfológicas y dimensionales de los predios dificultaron la reconversión, debido a que la forma y ubicación de los terrenos arrojaron superficies que por distanciamientos o rasantes produjeron polígonos irregulares inadecuados para acoger funcionalidades tipo, por lo que se ocuparon parcialmente y para destinos exclusivos, superando la dificultad desde la identificación e instalación del destino adecuado que no solo se adaptó a las condiciones sino que además potenció el uso, evidenciando que siempre existe la posibilidad de generar productos ambientalmente sostenibles y financieramente rentables, porque lo relevante es compatibilizar demanda y oportunidad, generando beneficios a sostenedores, operadores y ciudadanos que pueden acceder a la mejor oportunidad para la satisfacción de sus necesidades.

Un buen ejemplo de esta situación es el cambio de destino habitacional a sala cuna y jardín infantil ocurrido en el predio ubicado en calle Flor de Azucenas nro. 145 esquina Los Militares, en que en una superficie predial de 464,16 $\mathrm{m}^{2}$ se adaptó y amplió la superficie construida ${ }^{29}$ a 267,09 $\mathrm{m}^{2}$ para acoger a 38 lactantes y 30 párvulos, proyecto en que se optimizó el uso de los recursos aumentando la dotación del servicio en un área de dos kiló- metros cuadrados en que solo existían otras dos ofertas, las que eran insuficientes para atender a la población del sector, que en el último tiempo aumentó debido a la edificación en altura

La aplicación más exigente de la normativa ha sido la de obra nueva, aparentemente debido a que los predios factibles de desarrollar no parecieron atractivos a la industria porque la superficie factible de producir es muy inferior a la de la zona y los precios del suelo son semejantes o incluso superiores, por lo que al replicar la oferta existente la rentabilidad es inferior a la del sector, independiente de que la velocidad de venta debido al tamaño de los proyectos puede ser menor; análisis preliminar que no solo desmotivó la inversión sino que durante los primeros años de aplicación de la normativa no despertó interés. Situación distinta a la que ocurrió con el cambio de destino, que se entendió casi como la única opción para superar la pérdida de deseo por residir en una "casa isla" entendida como una externalidad difícil de transformar.

A la fecha los casos documentados son pocos, pero para sus respectivos nichos han sido emblemáticos, fundamentalmente porque al igual que en la opción "cambio de destino" ha habido un máximo de aprovechamiento de las posibilidades que la

29 La vivienda original tenía una superficie de $117,65 \mathrm{~m}^{2}$ en primer piso y según Permiso de Edificación $N^{\circ} 019$ de fecha 16 de febrero de 2011 se amplió en 149,44 $\mathrm{m}^{2}$ distribuidos en $46,63 \mathrm{~m}^{2}$ en el primer piso y $102,61 \mathrm{~m}^{2}$ en el segundo piso. 
FIGURA 2: EDIFICIO DE OFICINA DE SERVICIOS PROFESIONALES UBICADO EN CALLE HENDAYA NR0.263.

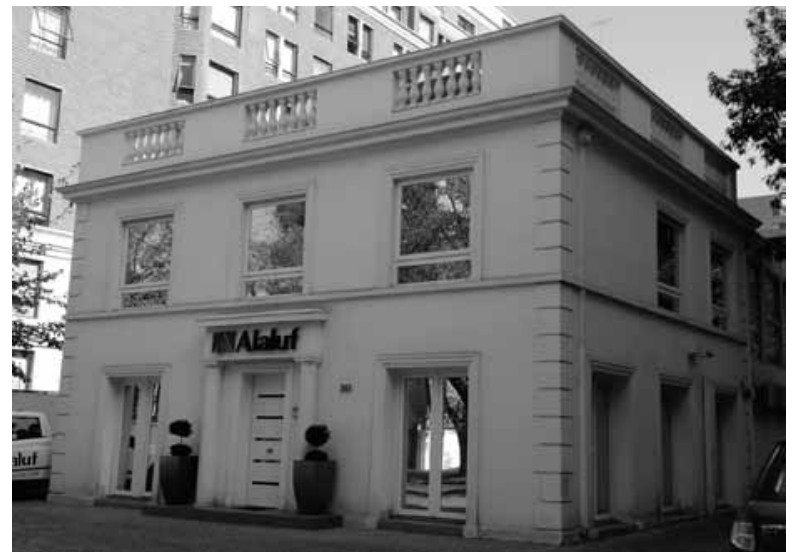

Fuente: Los autores, año 2015.

\section{FIGURA 4: SALÓN DE BELLEZA Y PELUQUERÍA} UBICADA EN CALLE NAPOLEÓN NR0.3375.

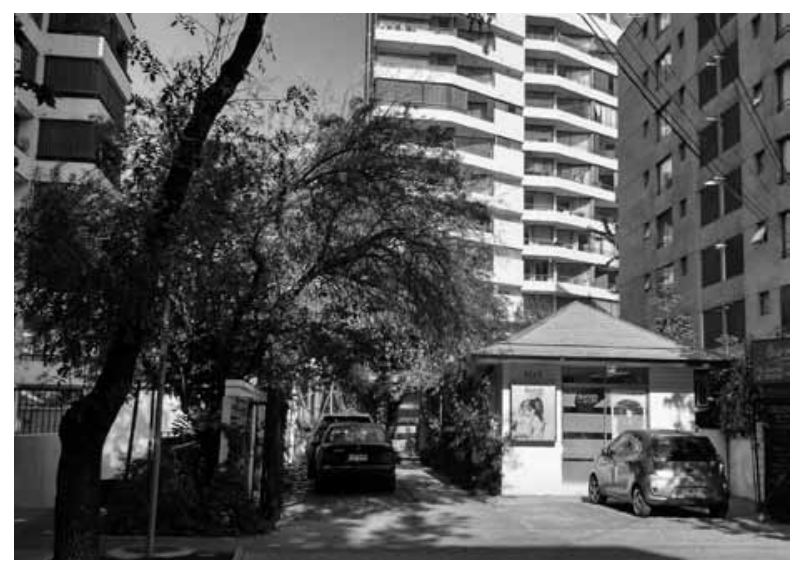

Fuente: Los autores, año 2015.
FIGURA 3: EDIFICIO DE EDUCACIÓN SUPERIOR, ESCUELA DE COCINA EN AVDA. AMÉRICO VESPUCIO SUR NR0.930.

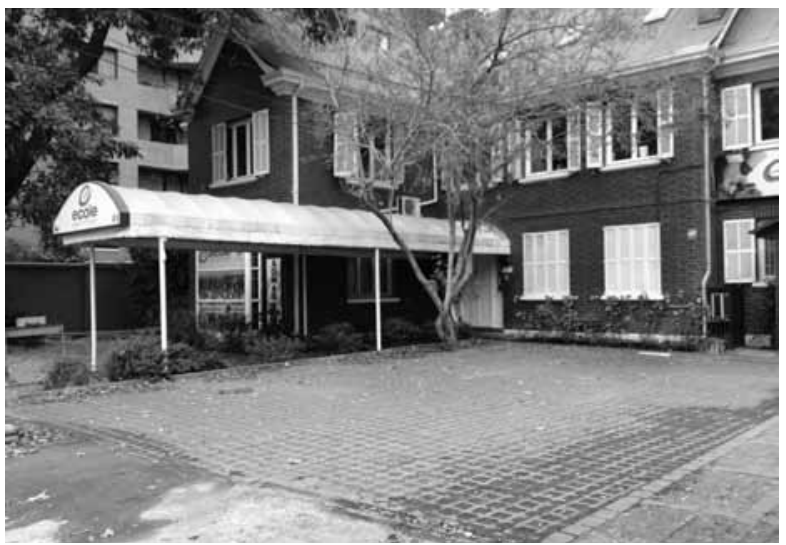

Fuente: Los autores, año 2015

FIGURA 5: SALA CUNA Y JARDÍN INFANTIL UBICADO EN CALLE FLOR DE AZUCENAS NRO.145.

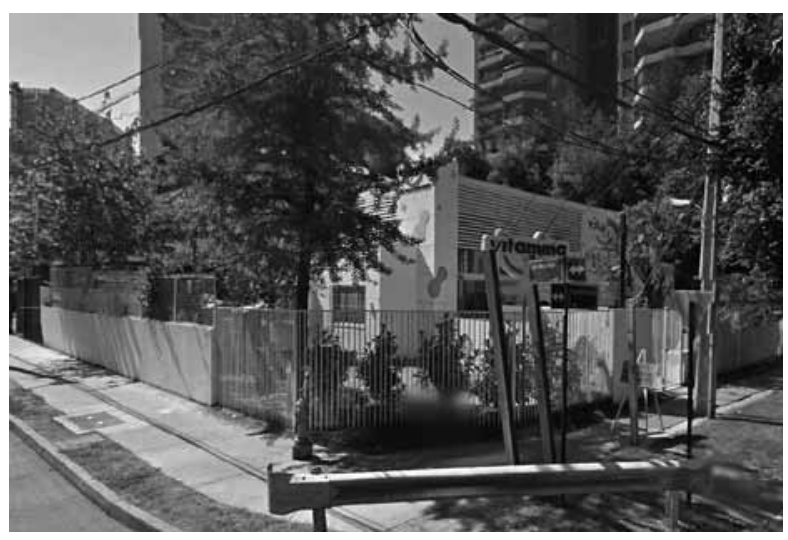

Fuente: Los autores, año 2014. 


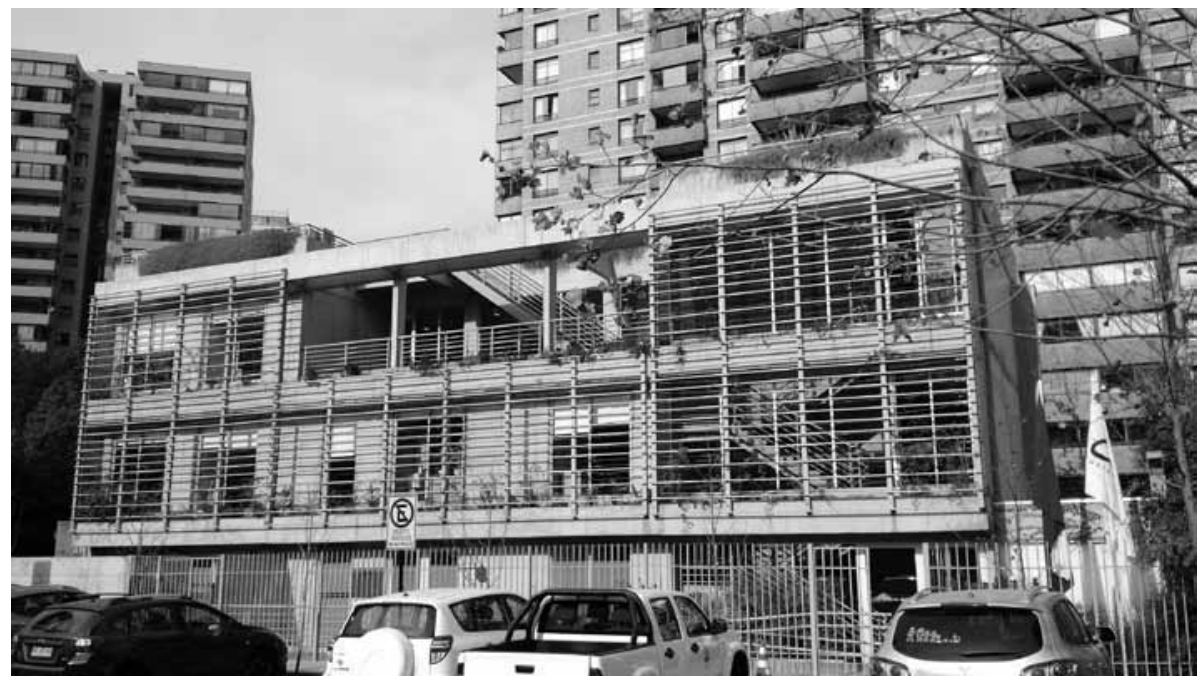

Fuente: Los Autores, año 2015.

normativa permite, especialmente enfocadas en la exclusividad de proponer opciones diferentes, que explotan valores desconocidos y presumiblemente deseados; lo que se tradujo en la búsqueda de soluciones de localización, espaciales, programáticas y constructivas innovadoras y por consiguiente captadoras de demandas insatisfechas y muchas veces ocultas, que en una primera aproximación destacan el destino y posteriormente la especificidad que aportan.

En el sector equipamiento y en consideración a la multiplicidad de opciones lo más importante para la identificación del destino y uso fue la relación entre la prestación y el lugar en que se entregó, lo que se observó en los casos levantados entre el año 2008 y el primer semestre 2015: el primero con Permiso de Edificación nro.056 de fecha 6 de marzo del año 2008 fue técnicamente una alteración que a consecuencia del tratamiento exterior se asemejó a una obra nueva, o al menos se leyó como tal, se trata de un predio de $646 \mathrm{~m}^{2}$ que cambió destino de "comercio y educación" a "servicios profesionales" en la calle La Pastora nro.128 alojando una sucursal bancaria en 435,53 $\mathrm{m}^{2}$ distribuidos en tres niveles. 
Las dos obras nuevas documentadas presentaron significativas improntas: la primera destinada a centro social fue construida por la Ilustre Municipalidad de Las Condes en un predio de 850,50 $\mathrm{m}^{2}$ ubicado en Avda. Alonso de Córdova (interior) con 904,38 $\mathrm{m}^{2}$ construidos distribuidos en tres niveles $^{30}$, acogió actividades que pudieron instalarse en ubicaciones de menor valor, sin embargo la decisión de instalación se entendió como un gesto hacia los habitantes de la comuna priorizando la localización de servicios en las mejores ubicaciones con calidad arquitectónica y constructiva. Adicionalmente es posible suponer que también fue una estrategia de incentivo al uso de la normativa. El segundo y no menos singular de los casos correspondió a la construcción de un edificio de tres pisos más subterráneo por un total de $416,36 \mathrm{~m}^{2}$ con solo $183,86 \mathrm{~m}^{2}$ sobre el nivel de suelo en un terreno de $334,85 \mathrm{~m}^{2}$ en calle Carmencita nro. $290^{31}$. Antes la propiedad había cambiado destino de habitacional a servicios profesionales, uso en el que la nueva obra insistió, demostrando que para la decisión de inversión lo más importante es el destino que asegura la mejor rentabilidad.

En la identificación del usuario de las edificaciones se observó que un porcentaje superior al 50\%

30 Permiso de edificación N052 de fecha 27 de marzo del año 2012. 31 Permiso de Edificación $N^{\circ} 086$ de fecha 23 de julio del 2013. corresponden a arrendamiento, demostrando que en el rubro comercial es frecuente la inversión en edificaciones para renta. Suelen ser desarrolladas por rentistas profesionales, como sociedades de inversión y aseguradoras o por personas naturales que intentan mejorar sus ingresos a través de la renta de un bien inmueble. Actividad que independiente de ser sujeto de tributos es una opción de rentabilidad de bajo riesgo, beneficiosa y eventualmente entendida como mitigadora de la pérdida de que fueron objeto los habitantes originales del sector.

Respecto de la producción residencial, el precedente fue el edificio ubicado en calle Glamis No3388 en que en un terreno de $720,68 \mathrm{~m}^{2}$ se construyó sobre nivel de suelo un edificio de cuatro pisos con $1.116,62 \mathrm{~m}^{2}$ con 16 departamentos y subterráneos con bodegas y estacionamientos ${ }^{32}$. El programa incluyó unidades de un nivel con un dormitorio de casi $40 \mathrm{~m}^{2}$ y unidades de dos niveles del tipo loft con patio privado de alrededor de $80 \mathrm{~m}^{2}$. El proyecto aprobado en Permiso de Edificación nro.165 de fecha 17 de junio del año 2003 acogió a la normativa de "predio remanente" establecido en el artículo 38 inciso cuarto de la Ordenanza del plan regulador de la comuna de Las Condes de octubre de

32 Balze, 2008. 
$1994^{33}$. Las ventas de las unidades fueron inscritas en el Conservador de Bienes Raíces entre fines del año 2004 y comienzos del año 2005, inmediatamente después de la recepción del edificio, lo que indicaría que todas las unidades fueron vendidas prácticamente durante el período de construcción lo que suele ser señal de una auspiciosa recepción de la oferta, que obedecería al precio inferior al de mercado, argumento con el que habitual y sesgadamente se explican las decisiones de consumo sin mayor profundización en razones que superan el precio y que se relacionan con las preferencias, el gusto, el deseo y lo más importante la satisfacción representada por la coincidencia entre las particularidades del producto ofertado y las expectativas de la demanda.

La velocidad de ventas del edificio de calle Glamis fue explicada por las características del producto que acogió una necesidad no cubierta de la población, que estuvo dispuesta a pagar incluso un valor superior al precio promedio del mercado de la zona por la exclusividad y probablemente futura plusvalía de la inversión ${ }^{34}$. La singularidad del producto no solo se focalizó en las unidades, sino

33 La normativa indicaba que en los predios remanentes localizados en zonas de alta y media densidad se podía aplicar una altura de cuatro pisos y catorce metros, 0,4 de ocupación de suelo y 1,6 de constructibilidad, además de distanciamientos de 4 metros y antejardín de 5 metros.

34 El seguimiento realizado a las transacciones de las unidades ocurridas desde la primera compra a la fecha, registró un incremento en el precio de hasta en un $10 \%$ por año. que también involucró los espacios comunes, que a diferencia de la oferta de la época los disminuyó, lo que podría explicarse por el tamaño y morfología del predio, pero que en definitiva resultó un atributo de valoración para un nicho que no demanda zonas comunes y tampoco está dispuesto a financiar su operación.

Los atributos de la propuesta edilicia se replicaron en un proyecto arquitectónica y programáticamente semejante que acogió a la normativa de "predio existente residual de densificación" aprobado en diciembre del año 2003, aumentando la altura de cuatro a cinco pisos con un máximo de 17,5 metros y la constructibilidad de 1,6 a 2,0. El permiso de edificación Nro.344 de fecha 21 de diciembre del 2004 autorizó en un terreno de 832,69 m² ubicado en calle Carmencita nro.262 la construcción de un edificio de $1.474,01 \mathrm{~m}^{2}$ sobre nivel de terreno con catorce departamentos de tipo loft distribuidos en cinco pisos, además de estacionamientos y bodegas en subterráneo. En la ejecución del proyecto hubo una serie de cambios $^{35}$ y discusiones respecto de la legitimidad de la propuesta, superadas las cuales el edificio fue recepcionado y vendido, y aunque

35 El proyecto registrado en Planos $\mathrm{N}^{\circ} 2034$ y N ${ }^{\circ} 2203 \mathrm{~A}$ inscritos en el Conservador de Bienes Raíces de Santiago indica superficie vendible $1.665,18 \mathrm{~m}^{2}$ en circunstancia que el 2,0 de constructibilidad era de $1.665,38 \mathrm{~m}^{2}$, distribuidos en 20 departamentos, todos con superficie inferior a $140 \mathrm{~m}^{2}$, altura inferior a $17,50 \mathrm{me}$ tros, nivel de ingreso bajo la cota 0,00 . 
probablemente debido a la contingencia los plazos de entrega aumentaron y los precios alcanzaron la media del mercado de la época, llama la atención el incremento del 10\% anual que ha tenido el precio de las segundas y terceras ventas ocurridas en nueve de los veinte casos, evidenciando la coincidencia de la propuesta con el interés de los demandantes, lo que instala como valor fundamental de cualquier oferta inmobiliaria la correspondencia entre la propuesta y las expectativas de la demanda, siendo fundamental en el diseño del producto la asertividad en la interpretación de las necesidades, especialmente las no cubiertas, intención que conlleva riesgo debido a que es difícil de detectar y también de financiar porque para la inversión lo más importante es la certidumbre.

Las dificultades jurídicas probablemente desmotivaron nuevas inversiones al ser percibidas como riesgosas, obviando el hecho de que la memoria inmobiliaria es frágil y siempre prioriza las conveniencias particulares representadas por la satisfacción de la adquisición del bien que cumple con las expectativas. La consecuencia fue que durante cuatro años no se registraron solicitudes de obra nueva que permitieran desarrollar predios residuales, que aumentaban sistemáticamente debido al desarrollo del proceso de densificación. Pareciera que la recepción y el beneficio que habían reportado las propuestas anteriores no motivó la inversión que privilegia la certidumbre, lo que sumado a que la magnitud de los proyectos y la disponibilidad de recursos obliga a evaluar ciclos cortos por lo que el riesgo aumenta, desincentivando la inversión. También es posible que la ausencia de proyectos de obra nueva obedeciera a la falta de interés de los sostenedores del dominio de los predios factibles de desarrollar, debido a la existencia de opciones alternativas y a la falta de propuestas atractivas. El último de los argumentos es técnicamente el más relevante y corresponde a las características de superficie y morfología de los predios residuales, que por variabilidad e irregularidad dificultan el diseño de las instalaciones que demandan los edificios residenciales. 
FIGURA 7: EDIFICIO CON DESTINO HABITACIONAL UBICADO EN CALLE CARMENCITA NR0.262.

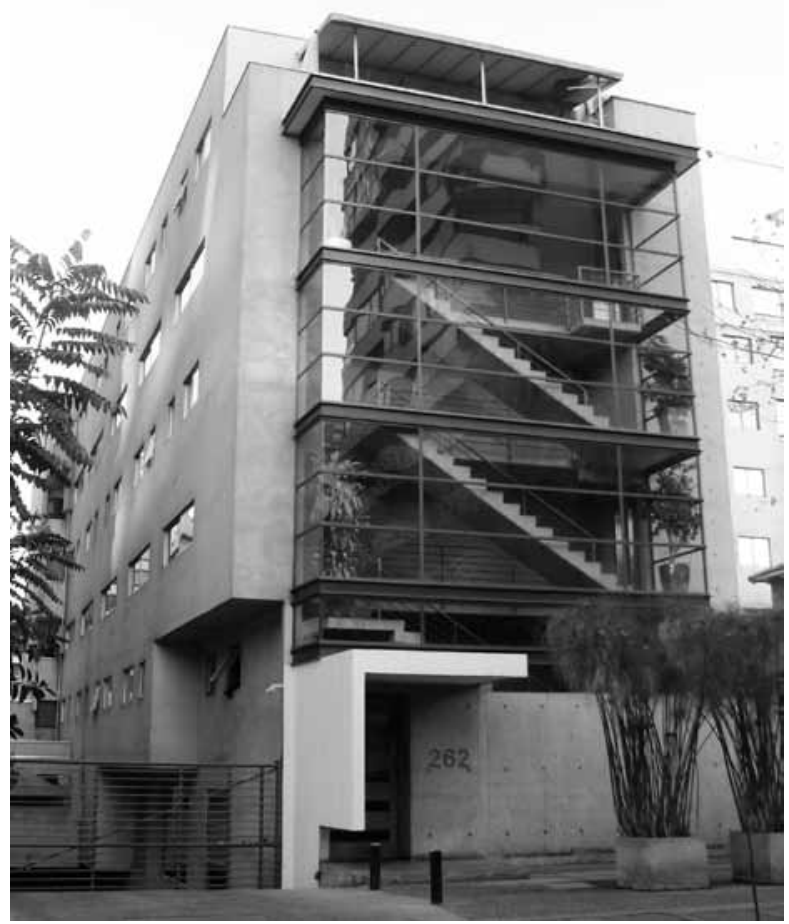

Fuente; Los autores, año 2015.
FIGURA 8: EDIFICIO CON DESTINO HABITACIONAL UBICADO EN AVDA, PRESIDENTE RIESCO NRO.3619.

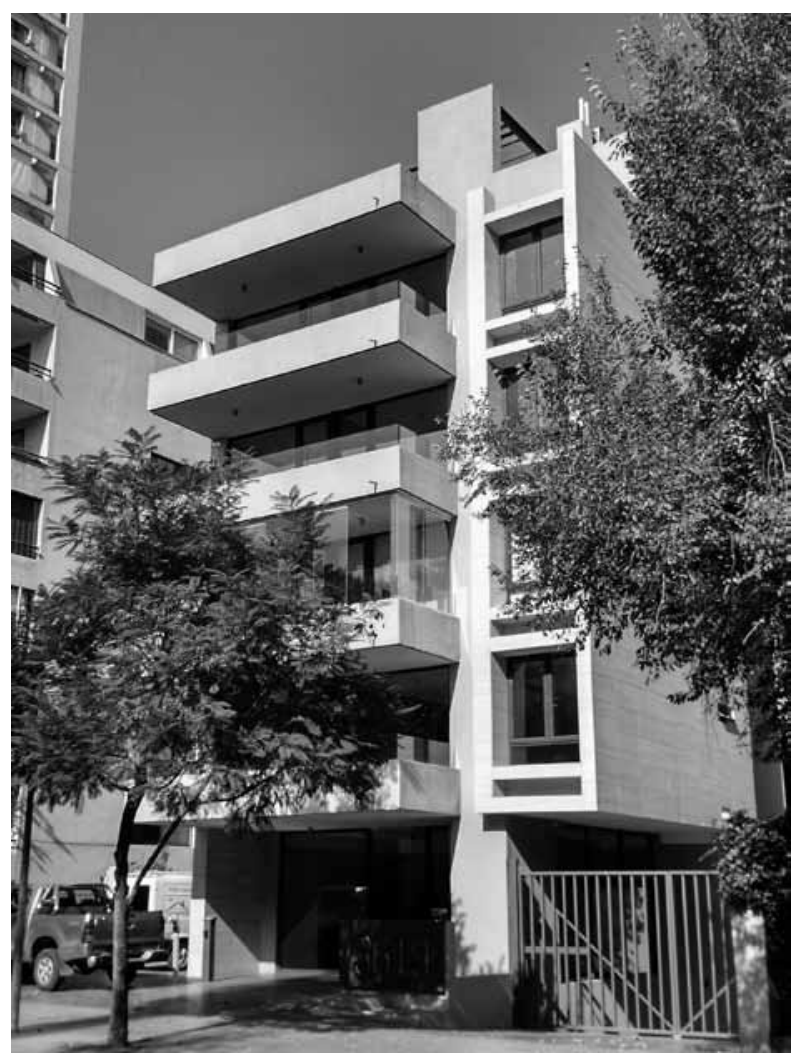

Fuente: Los autores, año 2015. 
Recién el 31 de diciembre del año 2010 se aprobó el siguiente proyecto que se acogió a la normativa, corresponde al Permiso de Edificación nro.115 que autorizó la construcción sobre terreno de 2.676,62 $\mathrm{m}^{2}$ en un predio de $1.030,05 \mathrm{~m}^{2}$ ubicado en la calle Versalles a consecuencia de la fusión de los terrenos nro.3005, nro.2997 y nro.2995. El proyecto propuso veinticinco viviendas de uno y dos dormitorios y tres locales comerciales. Las ventas fueron inscritas el año 2013 en el Conservador de Bienes Raíces y a la fecha se explota como hotel. Los otros cuatro proyectos de obra nueva con destino habitacional fueron autorizados entre los años 2012 y 2014, están localizados en: Piacenza nro.1135, Martín Alonso Pinzón nro.6914 y Domingo Bondi nro.1190 se encuentran en proceso de construcción y el último ubicado en Avda. Presidente Riesco nro.3619 está terminado y en proceso de venta. Según la empresa comercializadora con solo dos de veinte unidades disponibles, y aunque al mes de abril del año 2015 no se registraba inscripción en el Conservador de Bienes Raíces, pareciera que ha sido un producto exitoso, con algunas unidades ofertadas en renta a precios muy superiores a los del mercado de la zona.
En los proyectos se propusieron entre 16 y 25 unidades con superficies y programas variados, destacándose la incorporación de unidades de 214,96 $\mathrm{m}^{2}$. Respecto de las características espaciales de la oferta destacar el uso de la altura que permite la normativa y que posibilita la producción de viviendas con una mayor distancia a la habitual de piso a cielo que potencia espacialidades diferentes y absolutamente exclusivas, estrategia de exclusividad en el diseño que también se observó en detalles como la cantidad y dimensión de los estacionamientos ofertados que soportan automóviles de mayor tamaño. Los precios de venta por unidad de superficie han sido superiores a los del mercado, evidenciando en alguna medida que la valoración de los productos se identifica con el sector al que se pretende satisfacer.

La superficie de los predios utilizados varió entre $867,52 \mathrm{~m}^{2}$ y 1.316,64 m² y la morfología fue regular, lo que resulta significativo, porque aparentemente una de las mayores dificultades para el desarrollo de la arquitectura de proyectos habitacionales son los patrones y estándares normativos y culturales que deben combinarse dimensionalmente a fin de ocupar adecuadamente la superficie disponible, situación que la morfología predial suele dificultar. 


\section{FIGURA 9: EDIFICIO CON DESTINO HABITACIONAL UBICADO EN CALLE MARTÍN ALONSO PINZÓN NRO. 6914}

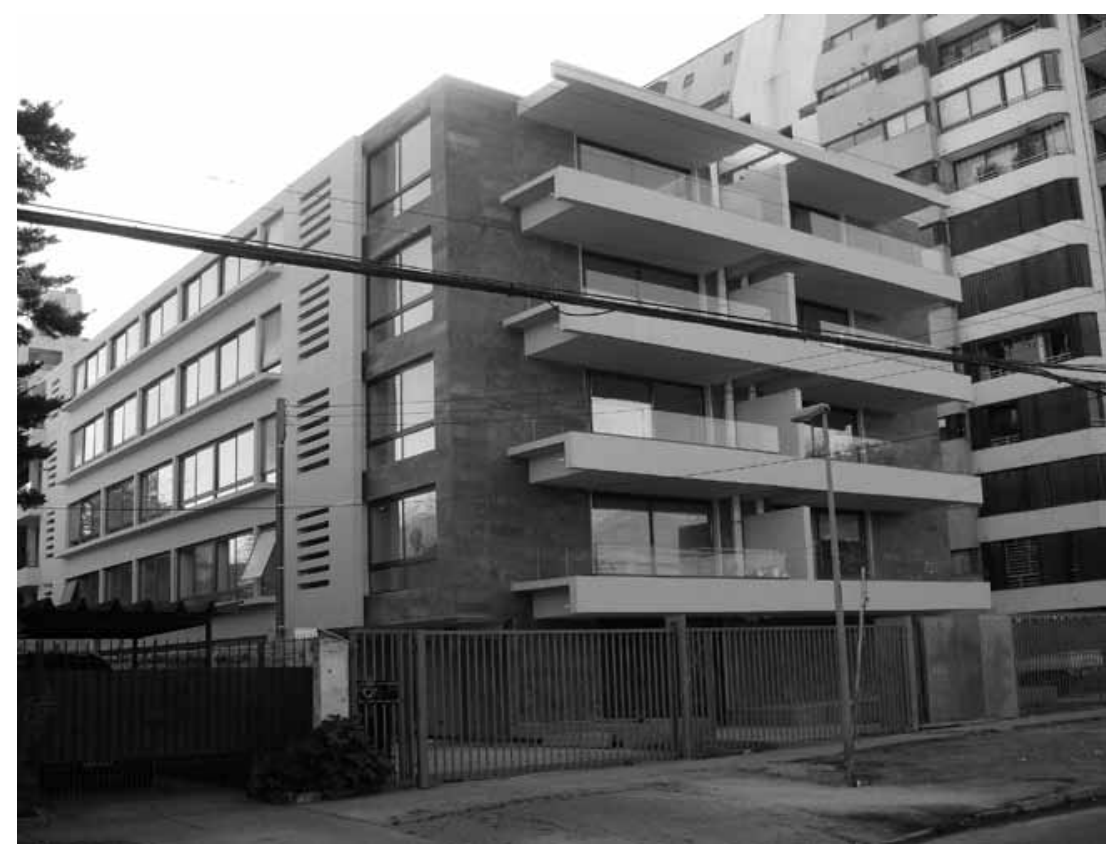

Fuente: Los autores, año 2015.

En síntesis, en los proyectos desarrollados se observó la búsqueda por nuevas y particulares opciones que acogen inquietudes y necesidades no resueltas en sectores con oferta homogénea, que sirviendo a la mayoría no asume sensibilidades distintas, habitualmente representadas por segmentos etarios y composiciones familiares distintas a los estándares, que por ser minoritarias constituyen un sector necesario de cubrir. Oportunidad que otorga la potencial producción de predios de menor dimensión y por consiguiente menor tamaño de proyecto, aparentemente menos intensiva en el uso de recursos y con menor tiempo de comercialización por lo que el éxito representado por la transferencia ocurre cuando se logran identificar los nichos de demanda. 
FIGURA NR0.10: MATRIZ DE CÁLCULO DE CABIDA SEGÚN SEGMENTACIÓN DE SUPERFICIE PREDIAL

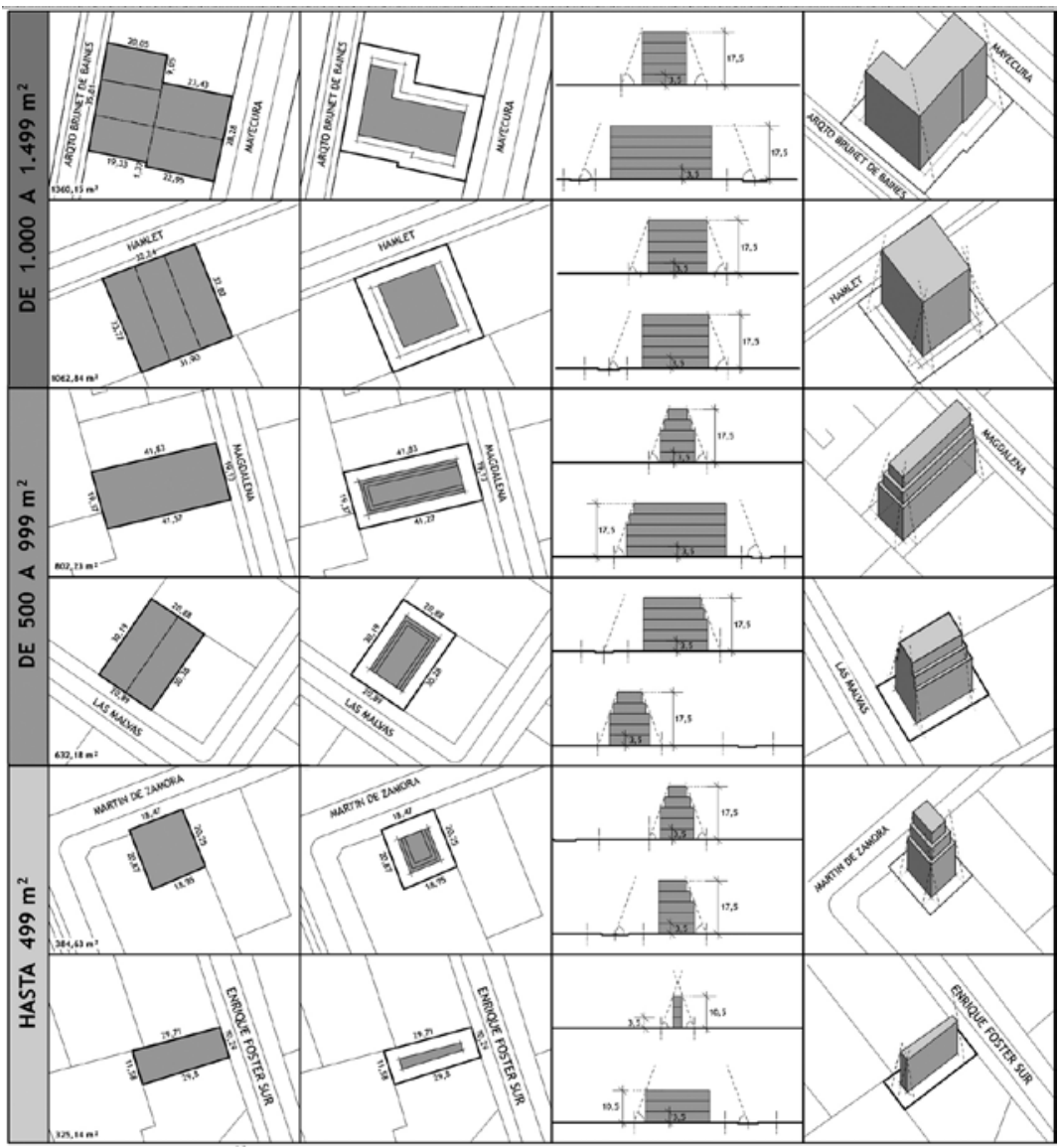

Fuente: Los autores, año 2014. 


\section{Conclusiones. Oportunidades para el desarrollo de nuevos predios que acojan la normativa}

La identificación de las posibilidades de desarrollo para los 56 dominios detectados a enero del año 2015 permitió caracterizar cada caso desde la situación, localización, posibilidades de fusión, superficie, morfología, cabida y entorno, información con la que se construyó una matriz que para cada dominio recomendó destino y potencial retorno. En el análisis se listaron oportunidades para distintos perfiles de emprendimientos y de emprendedores, también se identificó como posible mejora a la normativa el registro anticipado de la generación de terreno isla.

Se observó que alrededor del $20 \%$ de los dominios han cambiado informalmente de destino y que existen nueve potenciales fusiones que generarían superficies inferiores a $1.500 \mathrm{~m}^{2}$ reduciendo los casos a 35 . El $24 \%$ son predios de menos de $500 \mathrm{~m}^{2}$, el $58 \%$ entre 500 y $999 \mathrm{~m}^{2}$ y el $18 \%$ entre 1.000 y $1.499,99 \mathrm{~m}^{2}$. Mayoritariamente presentan buen estado de conservación e incluyen ambientaciones que intentan generar privacidad. El 50\% son arrendatarios, los casos de abandono o deterioro son escasos y habitualmente consecuencia de conflictos legales.
El estudio de cambio de destino y obra nueva para cada predio permitió identificar potenciales acciones de densificación en función de la superficie, espacialidad y factibilidad de reconversión, y del cálculo de la volumetría factible de desarrollar. El análisis de mercado y particularmente del entorno permitió listar potenciales usos, destacándose para obra nueva exclusivamente el destino habitacional y para cambio de destino distintas alternativas de equipamiento de alcance local porque los predios se localizan en zonas residenciales con ubicación interior respecto de las vías principales ${ }^{36}$.

Se desestimó la opción de obra nueva en los predios con superficie inferior a $500 \mathrm{~m}^{2}$ debido a que el volumen factible de producir no permite instalar unidades residenciales que cumplan con las condiciones normativas que permitan la operación, resultando mejor opción la reconversión que considera el aporte estructural y funcional de las edificaciones existentes, priorizándose los usos que requieran la menor cantidad de intervenciones y la mejor opción de rentabilidad, debiendo calcularse el tamaño del proyecto, para cuantificar inversiones, estimar costos e ingresos, calcular la recuperación y la rentabilidad. Las mejores opciones fueron jardines infantiles, locales de servicio de comida a domicilio y salones de belleza, peluquería y spa.

36 Pallarés, Lou y Pallarés, 2015. 
Para el resto de los predios ambas opciones resultaron factibles, por lo que resulta recomendable escoger en función de la disponibilidad de inversión, considerando que la mejor opción es la obra nueva habitacional que incluye variables de diseño que aportan a la diferenciación y potenciación de atributos de espacialidad y calidad, características posibles de abordar en proyectos de pocas unidades localizados en sectores valorados. La opción de cambio de destino es recomendable en los casos de estructuras de valor arquitectónico y espacial con morfologías factibles de transformar sin alterar la impronta, resultando particularmente atractivas las que presentan ocupación de suelo superior a la normativa actual.

En síntesis, la normativa de predio existente residual de densificación es aplicable y reporta beneficios para la ciudad al reconstruir el tejido urbano y para los sostenedores del dominio en cuanto les permite desarrollar sus propiedades en opciones que les reportan beneficios pecuniarios, sin embargo, y debido a que para un sector de la población la densificación siempre será motivo de expulsión, se recomienda incorporar en la normativa actual como condición de fusión de predios el desistimiento notarial de participación de los propietarios de los potenciales terrenos islas, a fin de que las decisiones y sus repercusiones estén informadas y puedan ser aceptadas.

\section{Bibliografía}

BALZE, Marianne. Edificio Loft El Golf: Las Condes, Chile. [En línea]. ARQ (Santiago). (69): 54-57, 2008. ISSN 0717-6996. DOI 10.4067/ S0717-69962008000200010.

BALLÉN ZAMORA, Sergio Alfonso. Vivienda social en altura. Antecedentes y características de producción en Bogotá. Revista INVI. 24(67): 95-124, noviembre 2009. ISSN 0718-8358. DOI 10.4067/ S0718-83582009000300004.

BARNETT, Jonathan. An introduction to urban design. New York, Harper \& Row. 1982. 260 p. ISBN 0-06-430376-4.

CHAVOYA GAMA, Jorge Ignacio; GARCíA GALVÁN, Joel y RENDÓN CONTRERAS, Héctor Javier. Una reflexión sobre el modelo urbano: ciudad dispersa-ciudad compacta. En: International Conference Virtual City and Territory (5 , 2009, Barcelona). Barcelona, Centre de Política de Sòl i Valoracions. 2009. p. 37-50.

GALETOVIC, Alexander, ed. Santiago, dónde estamos y hacia dónde vamos. Santiago, Centro de Estudios Públicos CEP. 2006. 579 p. ISBN 956-7015-39-2.

LARREA, Consuelo. Análisis de rentabilidad e inversión inmobiliaria en las principales comunas de Santiago. Santiago, Universidad de Chile, Universidad Politécnica de Madrid. 2005. Tesis Master en Dirección de Empresas Constructoras e Inmobiliarias. 
LÓPEZ, Ernesto; ARRIAGADA, Camilo; GASIK, Ivo y MEZA, Daniel. Efectos de la renovación urbana sobre la calidad de vida y perspectivas de relocalización residencial de habitantes centrales y peri centrales del área metropolitana del gran Santiago. EURE. 41(124): 45-67, septiembre 2015. ISSN 0250-7161. DOI 10.4067/ S0250-71612015000400003.

LÓPEZ DE LUCIO, Ramón. El espacio público en la ciudad europea: entre la crisis y las iniciativas de recuperación. Implicaciones para Latinoamérica. Revista de occidente. (230-231): 105-121, julio2000. ISSN 0034-8635.

MARDONES, Roberto. ¡No en mi patio trasero!: el caso de la comunidad ecológica de Peñalolen. Iconos. Revista de Ciencias Sociales. (34): 139149, 2009. ISSN 1390-1249. DOI 10.17141/ iconos.34.2009.331.

MARTIN, Barry y LERMAN, Philip. Under one roof: lessons I learned from a tough old woman in a little old house. New York, St. Martin's Press. 2013. 272 p. ISBN 978-1-4668-3913-7.

MARTÍNEZ LEMOINE, Rene. Densificar Santiago. Una receta equivocada. DUEP Diseño urbano y paisaje. 3(8), 2006. ISSN 0717- 9758.

PETERMANN, Alexandra. ¿Quién extendió Santiago? Una breve historia del límite urbano, 1953-1994. En: GALETOVIC, Alexander, ed. Santiago, dónde estamos y hacia dónde vamos. Santiago, Centro de Estudios Públicos CEP. 2006. p. 205-230. ISBN 956-7015-39-2.
PALLARÉS, Mirtha. La arquitectura religiosa en Santiago de Chile 1850-1950. Razones de las reminiscencias góticas. Madrid, Universidad Politécnica de Madrid. 2015. Tesis doctoral.

PALLARÉS, María Eugenia; LOU, Jing Chang y PALLARÉS, Mirtha. Aporte de la normativa especial de predios existentes residuales de densificación a la consolidación del tejido urbano. En: Congreso Arquisur (19, La Plata, Argentina 2015). Ciudades vulnerables. Proyecto o incertidumbre. La Plata, Universidad Nacional de La Plata. 2015. ISSN 2469-1305.

RAMÍREZ VIVEROS, José Miguel. La política pública de participación ciudadana en el gobierno local: caso Alcobendas, España. CONfines de relaciones internacionales y ciencia política. 8(15), 2012. ISSN 1870-3569.

SOTO, Nicolás y HORMAZÁBAL, Rodrigo. Mi vecino apesta: Una aproximación sociológica al fenómeno de LULU. Revista INVI. 24(65): 139-176, mayo 2009. ISSN 0718-8358. DOI 10.4067/ S0718-83582009000100005. 